\title{
Processo de semeadura e o rendimento do milho na região dos Campos Gerais do Paraná
}

\author{
Seeding process and corn yield in the region of Campos Gerais, State of Paraná, Brazil
}

\author{
Marcelo José Colet ${ }^{\mathrm{I}}$ Pedro Henrique Weirich Neto ${ }^{\mathrm{II}}$ Paulo William Garbuio ${ }^{\mathrm{III}}$ \\ Adriana Schimandeiro ${ }^{\mathrm{IV}}$
}

\section{RESUMO}

Na região dos Campos Gerais (PR), a cultura do milho ocupa aproximadamente um terço das áreas sob plantio direto no verão. Visando a manejos diferenciados, faz-se necessário conhecer as variáveis que influenciam no rendimento do milho. Este trabalho teve como objetivo correlacionar variáveis químicas e físicas de solo e antrópicas com a resposta da cultura do milho, considerando-se variabilidade espacial regional. As variáveis antrópicas estudadas foram: pressão de população, índice de pressão populacional, profundidade de semeadura e desvio padrão da profundidade de semeadura. As variáveis de solo estudadas foram: profundidade do horizonte A, areia, silte, argila, matéria orgânica, $\mathrm{pH}$, boro e manganês para a profundidade de $0-20 \mathrm{~cm}$, e areia, silte, argila, matéria orgânica, manganês e cobre para a profundidade $20-40 \mathrm{~cm}$. Para representar o rendimento da cultura, a variável resposta avaliada foi a massa de grãos da espiga por planta. Para redução do número de variáveis, utilizaram-se os métodos estatísticos de redução de variáveis explicativas "Todas Regressões Possíveis" (Best subsets) e "Passo-a-Passo" (Stepwise). As variáveis de solo reafirmaram-se como importantes para explicar o fenômeno massa de grãos. A variável antrópica profundidade de semeadura mostrou-se explicativa para o fenômeno estudado, demonstrando a influência do processo de semeadura no rendimento do milho.

Palavras-chave: plantio direto, semeadora, agricultura de precisão.

\section{ABSTRACT}

In the region of Campos Gerais, Paraná, Brazil, corn fields cover about one third of no-tillage summer cropland.
Before introducing alternative forms of management, it is necessary to gather information on variables that influence crop yield. This paper was aimed at correlating corn yield with chemical and physical soil variables, and human variables, considering spatial variability in the region. The human variables considered in the study were population pressure, population pressure index, seeding depth and standard deviation of seeding depth. Soil variables for the depth of $0-20 \mathrm{~cm}$ were thickness of horizon A, sand, silt, clay, organic matter, $\mathrm{pH}$, boron and manganese. For the depth of $20-40 \mathrm{~cm}$ were sand, silt, clay, organic matter, manganese, and copper. The variable mass of grains per plant represented crop yield. The statistical methods 'Best subsets' and 'Stepwise' were used to reduce the number of explanatory variables. Soil variables were reaffirmed as explanatory of mass of grains. The human variable seeding depth influences corn yield, showing the importance of the planting process on crop yield.

Key words: no tillage, seeder, precision farming.

\section{INTRODUÇÃO}

Mesmo sendo uma proposta de visão holística, é comum em trabalhos de agricultura de precisão a tentativa, pura e simples, de correlação entre a variabilidade de variáveis de fertilidade química de solo e o fenômeno rendimento das culturas. Vários autores correlacionaram o rendimento de culturas e algumas das variáveis físicas e/ou químicas do solo (MILLER et al., 1988 e FIORIN et al., 1997). Além de

\footnotetext{
IPrograma de Pós-graduação em Engenharia Agrícola, Universidade Estadual de Campinas (UNICAMP), Faculdade de Engenharia Agrícola (FEAGRI), Campinas, SP, Brasil. Fone: (19)9196.4648. E-mail: marcelo.colet@agr.unicamp.br. Autor para correspondência.

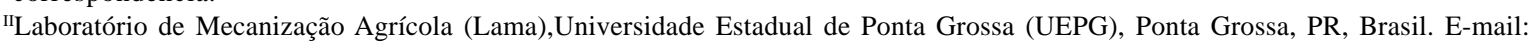
lama1@uepg.br.

IIIPrograma de Pós-graduação em Agronomia da Universidade Estadual de Ponta Grossa, Ponta Grossa, PR, Brasil.

${ }^{\text {IV }}$ Programa de Pós-graduação em Manejo Sustentável, Universidade Tecnológica de Munique, Munique, Alemanha.
} 
correlacionarem variáveis químicas e físicas do solo com o rendimento, outros autores obtiveram bons resultados quando consideraram alguma forma de representar a topografia do terreno (BRUBAKER et al., 1993; TIMLIN et al., 1998).

Pouca ou nenhuma importância é dada para a variabilidade da ação antrópica junto ao rendimento das culturas quando a agricultura de precisão é a área de interesse. Porém, vários trabalhos relatam a importância das operações agrícolas junto aos processos produtivos. No caso do processo de semeadura, SATTLER et al. (1996) observaram a influência da velocidade de semeadura na distribuição de plantas. SANTOS et al. (2003) verificaram que a mesma também é influenciada pela relação de área entre sementes de milho e orifícios de disco dosador.

Estudando variáveis de influência no rendimento do milho em uma lavoura comercial sob plantio direto, WEIRICH NETO (2004) ressalta a importância do processo de semeadura, responsável pela distribuição horizontal e vertical de sementes no solo. O aumento da população de plantas pode ser alternativa interessante para obtenção de rendimentos elevados (ALMEIDA et al., 2000). MEROTTO JUNIOR et al. (1999) verificaram a influência da uniformidade de emergência no número de grãos por espiga e na massa de grãos de milho.

Em trabalho em várias lavouras, GARBUIO (2002) qualificou como importantes, para o rendimento do milho, as variáveis convencionais de textura (areia e argila), o manganês e o teor de matéria orgânica, nas profundidades de $0-20 \mathrm{~cm}$ e $20-40 \mathrm{~cm}$; o boro e o $\mathrm{pH}$ na profundidade de $0-20 \mathrm{~cm}$ e o cobre na profundidade de 20-40cm. A análise de conjuntos compostos por grande número de variáveis apresenta algumas dificuldades. HAIR JUNIOR et al. (1998) discutem métodos estatísticos que permitam identificar as variáveis que possam explicar determinados fenômenos.

Este trabalho teve como objetivo correlacionar variáveis de solo já qualificadas por GARBUIO (2002) como explicativas para o fenômeno massa de grãos, bem como as antrópicas, representativas do processo de semeadura, com o rendimento da cultura do milho na região dos Campos Gerais do Paraná.

\section{MATERIAL E MÉTODOS}

O presente trabalho foi desenvolvido no ano agrícola 2001/2002. As 25 áreas agrícolas avaliadas estão situadas na região dos Campos Gerais e sua escolha baseou-se na distribuição espacial, bem como no cultivo de um dos dois híbridos de milho mais utilizados na região (TORK ${ }^{\circledR}$ - marca registrada Syngenta - e DKB $214^{\circledR}$ - marca registrada Dekalb). Todas as áreas encontravam-se sob sistema plantio direto. Em cada área acompanhou-se uma unidade amostral, composta por duas linhas de semeadura, sendo avaliadas dez plantas em cada uma das linhas da semeadora.

Dentre as variáveis de solo determinadas em laboratório após amostragem, consideraram-se apenas as variáveis de fertilidade física e química já destacadas por GARBUIO (2002) como de influência no rendimento de grãos da cultura do milho, para a região dos Campos Gerais. Para compor esta amostra, no ponto acompanhado de cada área agrícola, eram retiradas seis subamostras, quatro formando um quadrado imaginário na parte externa da unidade amostral e duas dentro desta. Embora alguns trabalhos discutam para o plantio direto a análise química do solo na profundidade de 0 a $10 \mathrm{~cm}$, optou-se por realizar as análises neste trabalho de 0 a $20 \mathrm{~cm}$ e de 20 a $40 \mathrm{~cm}$, pois a bibliografia existente neste caso é maior.

Como variáveis representativas da distribuição de plantas, determinou-se a pressão de população (PresPop) e o índice de pressão de população (IndPre). A pressão de população foi obtida multiplicando-se a soma das duas metades dos espaçamentos entre linhas, pela soma das metades dos espaçamentos de plantas nas linhas, obtendo-se assim a área individual teoricamente ocupada por cada planta, que, posteriormente, foi extrapolada para número de plantas por um hectare. O índice de pressão de população foi obtido pela razão da menor distância em relação às duas plantas vizinhas, pela maior distância, dentro de uma mesma linha, fornecendo assim um índice adimensional. Para tal, foram feitas duas medidas, uma com aproximadamente dez dias após a emergência das plantas e outra no momento da colheita, dando origem a quatro variáveis, duas para pressão de população, uma inicial e outra final (PresPIn e PresPFi), e duas para o índice de pressão populacional, também inicial e final (IndPrIn e IndPrFi)(SCHIMANDEIRO et al., 2006).

A determinação da profundidade média de semeadura (ProfMed) foi feita em todas as linhas amostradas, na parte externa da área amostral, observando-se uma distância de um metro como bordadura, aproximadamente 10 dias após o início da emergência. Amostraram-se de oito a dez plantas por linha, divididas entre os dois lados (anterior e posterior) da área amostral. A variável desvio da profundidade (DesvPr) foi obtida estatisticamente, pelo desvio padrão das amostras de profundidade.

Como variável resposta, utilizou-se a massa de grãos por planta, determinada para 10 plantas em 
cada linha de semeadura. A umidade da massa de grãos foi corrigida para $13 \%$.Os dados foram submetidos a teste de normalidade pelos métodos Anderson-Darling, Ryan-Joiner e Kolmogorov-Smirnov. Para as variáveis onde se verificou ausência de normalidade, transformaram-se os valores através de logaritmo neperiano (natural), raiz quadrada e inverso dos valores reais. Para a variável conteúdo de cobre no solo na camada de $20-40 \mathrm{~cm}$, devido à existência de valores entre 0 e $1 \mathrm{mg} \mathrm{dm}^{-3}$, multiplicou-se tais valores pela constante 5, transformando-se, em seguida, o resultado pelas funções matemáticas já descritas. De posse das variáveis geradas, variáveis originais transformadas, aplicaram-se novamente os testes citados para avaliação da normalidade, escolhendo-se a transformação que demonstrasse os maiores valores de probabilidade de normalidade. Após normalização, verificou-se a existência de correlação entre as variáveis independentes, sendo tal fenômeno denominado colinearidade e não é desejável, conforme HAIR JUNIOR et al. (1998). Para tal, mediu-se o nível de associação entre as variáveis pelo coeficiente de correlação (r). Quando duas ou mais variáveis apresentaram coeficiente de correlação igual ou superior a 0,8, manteve-se apenas uma variável na análise de regressão. Assim, as variáveis utilizadas foram o conteúdo de matéria orgânica determinado na profundidade de $0-20 \mathrm{~cm}$ (MO20), a argila de $0-20 \mathrm{~cm}$ (Arg20), o boro de $0-20 \mathrm{~cm}$ com transformação por logaritmo neperiano (lnB20), o manganês de $0-20 \mathrm{~cm}$ com transformação por logaritmo neperiano (lnMn20), o cobre de $20-40 \mathrm{~cm}$ com transformação por multiplicador 5 e logaritmo neperiano ( $\operatorname{lnCu} 40)$, o pH de $0-20 \mathrm{~cm}$ (pH20), a profundidade do horizonte A (ProfHzA), a pressão de população no florescimento (PrePopFi), o índice de pressão de população no florescimento (IndPreFi), a profundidade média (ProfMed) e o desvio da profundidade, transformado por logaritmo neperiano (lnDvPr).

Para a qualificação das variáveis junto ao fenômeno do rendimento do milho, utilizaram-se dois estudos estatísticos para a redução das variáveis explicativas, o método "passo a passo" (Stepwise) e o método “todas as regressões possíveis” (Best subsets), conforme HAIR JUNIOR et al. (1998).

Utilizaram-se quatro critérios para seleção dos melhores conjuntos regressores; o coeficiente de determinação $\left(\mathrm{R}^{2}\right)$, o coeficiente de determinação ajustado ( $\left.\mathrm{R}^{2} \mathrm{aj}\right)$, a estatística de Mallows (C-p) e a raiz quadrada do quadrado médio do resíduo (S), sendo este em relação à reta ajustada. Para os valores do coeficiente de determinação e determinação ajustado, estes variam de zero a um, sendo os valores mais próximos de um indicativos de um melhor ajuste entre a curva sugerida (no caso específico, pelo conjunto de variáveis explicativas sugeridas) e os valores reais do fenômeno. A utilização do coeficiente de determinação ajustado se faz interessante quando se têm conjuntos regressores com variáveis diferentes, porém com o mesmo número de variáveis regressoras. $\mathrm{O}$ valor da estatística de Mallows deve ser o mais próximo do número de variáveis regressoras no modelo. Para a raiz quadrada do quadrado médio do resíduo, quanto menor o valor, mais interessante é o modelo (HAIR JUNIOR et al., 1998).

O programa computacional utilizado para as análises foi o MINITAB $12.2^{\circledR}$ (Direitos reservados Minitab Inc.).

Para a caracterização climática do período, utilizaram-se dados referentes à precipitação e temperatura das estações meteorológicas de Ponta Grossa (2513' 29”'S e 5001'16”O) e de Telêmaco

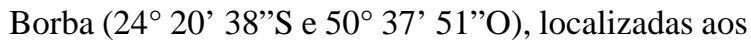
limites sul e norte da região de estudo, respectivamente.

\section{RESULTADOS E DISCUSSÃO}

A caracterização climática do período estudado, precipitação e temperatura, está representada na figura 1. Cabe citar que a época de semeadura para a maioria das áreas amostradas ocorreu a partir da segunda quinzena de setembro. Estudando a cultura do milho em Ponta Grossa (PR) a partir de uma série de 32 anos de dados climáticos, CARAMORI et al. (1991) identificaram probabilidade de ocorrência de déficit hídrico nos meses de novembro e dezembro, diminuindo a partir de janeiro. Conforme a figura 1, observam-se baixos valores de precipitação para a segunda quinzena do mês de novembro, antes do previsto pelos autores, porém em período mais curto. Segundo FANCELLI \& DOURADO-NETO (2000), tal condição poderia causar uma restrição ao desenvolvimento da cultura, coincidindo com a fase de desenvolvimento vegetativo. Para os mesmos, os valores de temperatura, também observados na figura 1, não são limitantes, caracterizando o ano agrícola como climaticamente favorável.

A estatística descritiva e testes de normalidade aplicada nas variáveis utilizadas na análise estão na tabela 1 . O coeficiente de variação apresentado para profundidade de semeadura aproxima-se do valor de $20 \%$, em ensaios de semeadoras em plantio direto, CASÃO JUNIOR et al. (1998) e CASÃO JUNIOR et al. (2000) encontraram CVs de 22,9 a 41,6\% e de 26,3 a $32,7 \%$ nas linhas para a profundidade de deposição de semente. Analisando duas linhas de uma semeadora, 


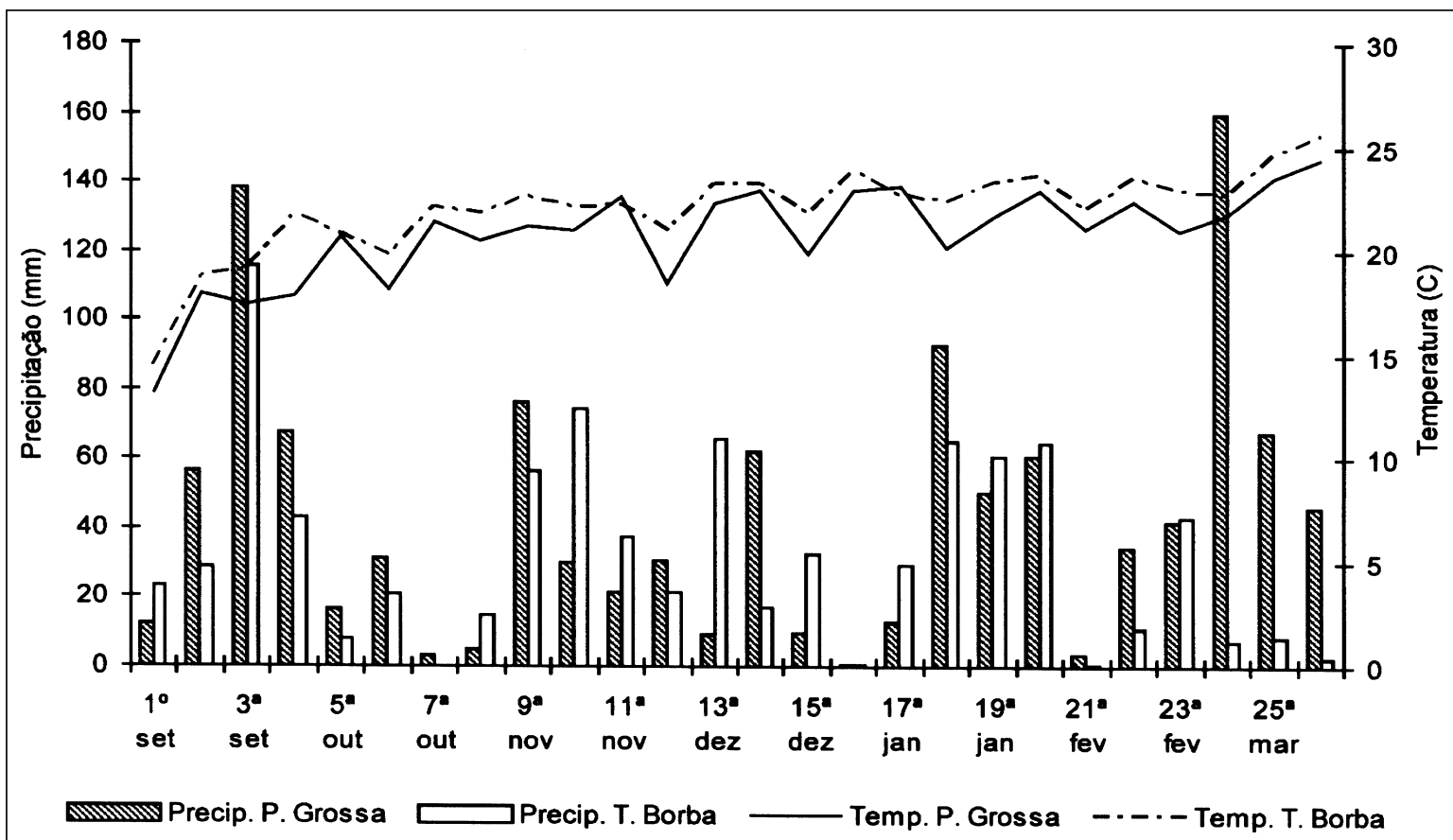

Figura 1 - Precipitação e temperatura semanais conforme as estações meteorológicas de Ponta Grossa e Telêmaco Borba.

WEIRICH NETO (2004) relata valores de 21,8 e 28,8\%.

Os conjuntos de variáveis explicativas, para a variável massa de grãos, selecionadas pelo método "todas as regressões possíveis" são apresentados na tabela 2. Apesar de o valor C-p do segundo conjunto de variáveis proposto por este método não ser o mais próximo ao número de variáveis incluídas no modelo, o mesmo apresenta o menor valor de $\mathrm{S}$ e a menor quantidade de variáveis incluídas, em relação aos conjuntos com melhor valor para o C-p. Sendo assim,

Tabela 1 - Análise descritiva e de normalidade das variáveis.

\begin{tabular}{|c|c|c|c|c|c|c|c|c|}
\hline \multirow[b]{2}{*}{ Variáveis* } & \multicolumn{5}{|c|}{ Análise descritiva } & \multicolumn{3}{|c|}{ Análise de normalidade } \\
\hline & Média & Desvio Padrão & $\mathrm{CV}(\%)$ & Máximo & Mínimo & $\begin{array}{c}\text { Anderson } \\
\text { Darling }\end{array}$ & $\begin{array}{l}\text { Ryan } \\
\text { Joiner }\end{array}$ & $\begin{array}{c}\text { Kolmogorov } \\
\text { Smirnov }\end{array}$ \\
\hline MO20 $\left(\mathrm{g} \mathrm{dm}^{-3}\right)$ & 43,1 & 11,2 & 27 & 60 & 19 & 0,500 & $>0,10$ & $>0,15$ \\
\hline pH20 & 4,9 & 0,28 & 6,6 & 5,4 & 4,5 & 0,220 & $>0,10$ & $>0,15$ \\
\hline $\operatorname{lnB} 20\left(\mathrm{mg} \mathrm{dm}^{-3}\right)$ & $-1,56$ & 0,28 & 18 & $-1,07$ & $-2,04$ & 0,281 & $>0,10$ & $>0,15$ \\
\hline $\operatorname{lnMn} 20\left(\mathrm{mg} \mathrm{dm}^{-3}\right)$ & 1,88 & 0,59 & 31 & 2,89 & 1,03 & 0,239 & $>0,10$ & $>0,15$ \\
\hline $\operatorname{lnCu} 40\left(\mathrm{mg} \mathrm{dm}^{-3}\right)$ & 1,98 & 0,78 & 39 & 3,49 & 0,0 & 0,235 & $>0,10$ & $>0,15$ \\
\hline $\operatorname{Arg} 20\left(\mathrm{~g} \mathrm{~kg}^{-1}\right)$ & 342,7 & 137,1 & 40 & 560 & 142 & 0,190 & $>0,10$ & $>0,15$ \\
\hline ProfHzA (mm) & 216,8 & 65,7 & 30 & 370,0 & 120,0 & 0,125 & $>0,10$ & $>0,15$ \\
\hline ProfMed (mm) & 45,3 & 10,5 & 23 & 60,0 & 22,0 & 0,244 & $>0,10$ & $>0,15$ \\
\hline lnDesvProf (mm) & 0,294 & 0,181 & 61 & 0,775 & 0,009 & 0,676 & $>0,10$ & $>0,15$ \\
\hline PresPIn (pl ha'-1) & 57240 & 12388 & 22 & 76509 & 35194 & 0,643 & $>0,10$ & $>0,15$ \\
\hline IndPreIn & 0,67 & 0,084 & 12 & 0,82 & 0,50 & 0,165 & $>0,10$ & 0,139 \\
\hline PresPFi (pl ha ${ }^{-1}$ ) & 56370 & 13672 & 24 & 76509 & 21781 & 0,745 & $>0,10$ & $>0,15$ \\
\hline IndPreFi & 0,664 & 0,095 & 14 & 0,82 & 0,42 & 0,299 & $>0,10$ & $>0,15$ \\
\hline
\end{tabular}

*Conteúdo de matéria orgânica do solo na camada de 0-20cm (MO), potencial hidrogênio iônico do solo de 0-20cm (pH20), logaritmo neperiano do conteúdo de Boro de $0-20 \mathrm{~cm}\left(\operatorname{lnB}_{2} 0\right)$, logaritmo neperiano do conteúdo de Manganês de 0-20cm (lnMn20), logaritmo neperiano do conteúdo de Cobre de 20-40 cm (lnCu40),conteúdo de argila de 0-20cm (Arg20), profundidade do horizonte A (ProfHzA), profundidade média de deposição da semente (ProfMed), logaritmo neperiano do desvio padrão da profundidade de deposição da semente (lnDesvProf), pressão de população inicial e final (PresPIn e PresPFi), índice de pressão de população inicial e final (IndPresIn e IndPresFi). 
Tabela 2 - Variáveis regressoras apontadas pelo método “todas regressões possíveis” para a variável dependente massa de grãos por espiga e valores dos critérios de seleção adotados: coeficiente de determinação $\left(R^{2}\right)$; coeficiente de determinação ajustado ( $\mathrm{R}^{2}$ aj); estatística de Mallows (C-p) e raiz quadrada do quadrado médio do resíduo (S).

\begin{tabular}{|c|c|c|c|c|c|c|c|c|c|c|c|c|c|c|}
\hline \multicolumn{4}{|c|}{ critérios de seleção } & \multicolumn{11}{|c|}{ variáveis regressoras* } \\
\hline $\mathrm{R}^{2}$ & $R^{2} a j$ & C-p & $S$ & $\begin{array}{c}\mathrm{MO} \\
20\end{array}$ & $\begin{array}{c}\text { Arg } \\
20\end{array}$ & $\begin{array}{c}\ln \\
\text { B20 }\end{array}$ & $\begin{array}{c}\ln \mathrm{Mn} \\
20\end{array}$ & $\begin{array}{c}\ln \\
\mathrm{Cu} 40\end{array}$ & pH 20 & $\begin{array}{l}\text { Prof } \\
\text { HzA }\end{array}$ & $\begin{array}{c}\text { Pre } \\
\text { PopFi }\end{array}$ & $\begin{array}{c}\text { Ind } \\
\text { Pre } \\
\text { Fi }\end{array}$ & $\begin{array}{c}\text { Pr } \\
\text { Med }\end{array}$ & $\begin{array}{c}\text { ln } \\
\text { Dv } \\
\text { Pr }\end{array}$ \\
\hline 0,69 & 0,58 & 3,9 & 14,58 & $\mathrm{x}$ & & $\mathrm{x}$ & $\mathrm{x}$ & & $\mathrm{x}$ & $\mathrm{x}$ & & & $\mathrm{x}$ & \\
\hline 0,71 & 0,59 & 4,8 & 14,47 & $X$ & $\mathrm{x}$ & & $\mathrm{x}$ & & $\mathrm{x}$ & $\mathrm{x}$ & & & $\mathrm{x}$ & $\mathrm{x}$ \\
\hline 0,72 & 0,58 & 6,4 & 14,65 & $\mathrm{x}$ & $\mathrm{x}$ & $\mathrm{x}$ & $\mathrm{x}$ & & $\mathrm{x}$ & $\mathrm{x}$ & & & $\mathrm{x}$ & $\mathrm{x}$ \\
\hline 0,72 & 0,56 & 8,1 & 14,97 & $\mathrm{x}$ & $\mathrm{x}$ & $\mathrm{x}$ & $\mathrm{x}$ & $\mathrm{x}$ & $\mathrm{x}$ & $\mathrm{x}$ & & & $\mathrm{x}$ & $\mathrm{x}$ \\
\hline 0,72 & 0,55 & 8,3 & 15,11 & $\mathrm{x}$ & $\mathrm{X}$ & $\mathrm{x}$ & $\mathrm{x}$ & & $\mathrm{x}$ & $\mathrm{x}$ & $\mathrm{x}$ & & $\mathrm{x}$ & $\mathrm{x}$ \\
\hline 0,73 & 0,53 & 10,0 & 15,47 & $\mathrm{x}$ & $\mathrm{x}$ & $\mathrm{x}$ & $\mathrm{x}$ & $\mathrm{x}$ & $\mathrm{x}$ & $\mathrm{x}$ & & $\mathrm{x}$ & $\mathrm{x}$ & $\mathrm{x}$ \\
\hline 0,73 & 0,53 & 10,0 & 15,47 & $\mathrm{x}$ & $\mathrm{X}$ & $\mathrm{x}$ & $\mathrm{x}$ & $\mathrm{x}$ & $\mathrm{X}$ & $\mathrm{x}$ & $\mathrm{x}$ & & $\mathrm{x}$ & $\mathrm{x}$ \\
\hline 0,73 & 0,49 & 12,0 & 16,04 & $\mathrm{x}$ & $\mathrm{X}$ & $\mathrm{x}$ & $\mathrm{x}$ & $\mathrm{x}$ & $\mathrm{x}$ & $\mathrm{x}$ & $\mathrm{x}$ & $\mathrm{x}$ & $\mathrm{x}$ & $\mathrm{x}$ \\
\hline
\end{tabular}

*Conteúdo de matéria orgânica de 0-20cm (MO20), conteúdo de argila de 0-20cm (Arg20), logaritmo neperiano do conteúdo de Boro de 0$20 \mathrm{~cm}\left(\operatorname{lnB}_{2} 0\right)$, logaritmo neperiano do conteúdo de Manganês de 0-20cm (lnMn20), logaritmo neperiano do conteúdo de Cobre de 20-40cm (lnCu40), potencial hidrogênio iônico do solo de $0-20 \mathrm{~cm}\left(\mathrm{pH}_{2} 0\right)$, profundidade do horizonte A do solo (ProfHzA), pressão de população final de plantas (PrePopFi), índice de pressão de população final de plantas (IndPreFi), profundidade de deposição da semente (PrMed) e logaritmo do desvio padrão da profundidade de deposição da semente (lnDvPr).

optou-se pela escolha do conjunto com as variáveis, matéria orgânica, argila, manganês e $\mathrm{pH}$, todos na profundidade de $0-200 \mathrm{~mm}$, bem como a profundidade do horizonte A, a profundidade de semeadura e o desvio da profundidade de semeadura.

Para as variáveis matéria orgânica $(0-20 \mathrm{~cm})$, qualificada pelos dois métodos estatísticos utilizados, e argila $(0-20 \mathrm{~cm})$, selecionada pelo método "todas as regressões possíveis”, os resultados obtidos corroboram os de LOPES \& COX (1979), enquanto MULLA \& SHEPERS (1997) classificam a disponibilidade de água, a profundidade de solum, disponibilidade de nutrientes, a textura, a matéria orgânica e o pH como as características mais importantes. FIORIN et al. (1997) indicaram correlação entre a profundidade de solum e o rendimento de culturas.

No caso do conteúdo de argila e da profundidade do horizonte $\mathrm{A}$, estas respostas são importantes devido à baixa variabilidade temporal das mesmas. A relação custo/benefício de uso destas variáveis como base do manejo localizado da fertilidade do solo pode ser bem interessante.

A inclusão das variáveis profundidade de semeadura e desvio da profundidade de semeadura denota a influência do processo de semeadura no rendimento da cultura. Ao contrário do estudo de WEIRICH NETO (2004), as variáveis pressão de população e índice de pressão de população não se mostraram importantes. Vários trabalhos descreveram alguma forma de correlação entre o rendimento de milho e a heterogeneidade de emergência (NAFZIGER et al.,
1991; FORD \& HICKS, 1992). Problemas de vigor das plântulas, incidência de pragas e doenças na fase inicial e má polinização podem ser catalisados pela variabilidade temporal de emergência de plântulas.

Dentro do processo de semeadura, LIU et al. (2004) relatam haver maior correlação do rendimento do milho com a variabilidade de emergência do que com a variabilidade de distribuição de plantas.

Neste caso, a importância do processo de semeadura pode denotar a qualidade do sistema de produção de milho regional, fazendo com que detalhes tidos como pouco importantes em um primeiro momento comecem a assumir importância, pois outros fatores, como a complementação química inicial, já foram superados.

Pelo método "passo-a-passo”, as variáveis selecionadas como explicativas, coeficiente de determinação $\left(R^{2}=0,68\right)$ e coeficiente de determinação ajustado $\left(R^{2} a j=0,59\right)$, foram a profundidade média de deposição de semente (ProfMed), o conteúdo de matéria orgânica na profundidade de $0-20 \mathrm{~cm}$ (MO20), o logaritmo neperiano do Manganês de $0-20 \mathrm{~cm}$ (lnMn20), a profundidade do horizonte A (ProfHzA) e o pH de $0-20 \mathrm{~cm}$ (pH20). Esta seleção difere das selecionadas pelo método "todas as regressões possíveis” por não incluir as variáveis argila $(0-20 \mathrm{~cm})$ e desvio da profundidade de semeadura.

Para os dois métodos utilizados na seleção de variáveis regressoras, os valores do coeficiente de determinação podem ser considerados razoáveis, considerando-se que o fenômeno estudado é biológico. 
Porém, a utilização destas variáveis no dimensionamento quantitativo do fenômeno rendimento de milho não seria interessante. Convém salientar que o objetivo deste trabalho é uma seleção qualitativa de variáveis, esperando utilizá-las no estudo de zonas de manejo diferenciado.

Comparando os teores de manganês apresentados na tabela 1 com os níveis propostos por RAIJ et al. (1997), são observados teores médios e altos para o nutriente em todas as áreas amostradas. A sua inclusão como variável explicativa para o fenômeno massa de grãos pode estar associada a sua interação com outros elementos presentes no solo.

\section{CONCLUSÕES}

Dentre as variáveis de solo sugeridas anteriormente, a matéria orgânica, o manganês e o pH na profundidade de $0-20 \mathrm{~cm}$ mostraram-se importantes à explicação do rendimento da massa de grãos. Das variáveis representantes do processo de semeadura, a profundidade de semeadura foi classificada como variável explicativa do fenômeno rendimento da massa de grãos. Quanto às características físicas do solo, a profundidade do horizonte A do solo também foi qualificada como explicativa pelos dois métodos de redução de variáveis, enquanto a argila foi classificada com explicativa por apenas um dos métodos.

\section{REFERÊNCIAS}

ALMEIDA, M.L. et al. Incremento na densidade de plantas: uma alternativa para aumentar o rendimento de grãos de milho em regiões de curta estação estival de crescimento. Ciên Rural, v.30, n.1, p.23-29, 2000.

BRUBAKER, S.C. et al. Soil properties associated with landscape position. Soil Sci Soc Am J, v.57, p.235-239, 1993.

CARAMORI. P.H. et. al. Frequência de ocorrência de períodos com deficiência hídrica (veranicos) no estado do Paraná. Londrina: IAPAR, 1991. 40p. (Boletim Técnico n. 36, março 1991).

CASÃO JUNIOR, R. et al. Avaliação do desempenho da semeadora-adubadora Magnum 285-PD. Londrina: IAPAR, 1998. 47p. (Circular 105).

CASÃO JUNIOR, R. et al. Desempenho da semeadoraadubadora AS 13500-Vence Tudo em solos argilosos. Londrina: IAPAR, 2000. 46p. (Circular 110).

FANCELli, A.L.; DOURADO-NETO, D. Produção de milho. Guaíba: Agropecuária, 2000. 360p.

FORD, J.H.; HICKS, D.R. Corn growth and yield in uneven emerging stand. J Prod Agric, v.5, n.9, p.185-189, 1992.
FIORIN, J.E. et al. Armazenamento de água no solo e crescimento e produção de milho. $\mathbf{R}$ bras Ci Solo, v.21, n.2, p.241-247, 1997.

GARBUIO, P.W. Correlação de variáveis químicas e físicas do solo com o rendimento da cultura do milho (Zea mays L.) considerando-se variabilidade espacial regional. 2002. 52f. Monografia (Curso de Agronomia) - Universidade Estadual de Ponta Grossa, Ponta Grossa.

HAIR JUNIOR, F. et al. Multivariate data analysis. Upper Saddle River: Prentice Hall, 1998. 730p.

LIU, W. et al. Response of corn grain yield to spatial and temporal variability in emergence. Crop Science. v.44, p.847854, 2004

LOPES, A.S.; COX, F.R. Relação de características físicas, químicas e mineralógicas com fixação de fósforo em solos sob cerrados. R bras Ci Solo, v.3, p.82-88, 1979.

MEROTO JUNIOR, A. et al. A desuniformidade de emergência reduz o rendimento do milho. Ciên Rural, v.29, n.4, p.595601, 1999.

MILLER, M.P. et al. Spatial variability of wheat yield and soil properties on complex hills. Soil Sci Soc Am J, v.52, p.11331141, 1988.

MULLA, D.J.; SCHEPERS, J.S. Key processes and propriertes for site-specific soil and crop management. In: PIERCE, F.J.; SADLER, E.J. The state of site-specific management for agriculture. Madison, WI: ASA Misc, ASA, CSSA e SSSA, 1997. p.1-18

NAFZIGER, E.D. et al. Response of corn uneven emergence. Crop Science, v.31, p.811-815, 1991.

RAIJ, B. van; et al. Recomendações de adubação e calagem para o Estado de São Paulo. 2.ed. Campinas: Instituto Agronômico: Fundação IAC, 1997. 285p. (Boletim Técnico, 100).

SANTOS, S.R. dos et al. Espaço livre entre orifícios de discos dosadores e sementes de milho na eficiência de semeadura. Enge Agríc, v.23, n.2, p.300-308, 2003.

SATTLER, A. et al. Avaliação do desempenho de semeadoras de precisão em plantio direto de milho. Rev Plan Dir, Passo Fundo, v.1, n.33, p.27, 1996.

TIMLIN, D.J. et al. Spatial and temporal variability of corn grain yield on a hillscope. Soil Sci Soc Am J, v.62, p.764773, 1998

SCHIMANDEIRO, A. et al. Distribuição longitudinal de plantas de milho (Zea mays L.) na região dos Campos Gerais, Paraná. Ciênc Rural, v.36, n.3, p.977-980, 2006

WEIRICH NETO, P.H. Importância de atributos agronômicos para qualificação da semeadura do Milho (Zea mays) no Sistema Plantio Direto na Região dos Campos Gerais - PR. 2004. 147f. Tese (Doutorado em Água e Solo) - Universidade Estadual de Campinas, Campinas, Faculdade de Engenharia Agrícola. 